\title{
DEHALOGENATION OF POLYCHLORINATED BIPHENYLS (PCB) BY NUCLEOFILE REACTANTS AT THE PRESENCE OF IONIC LIQUIDS AND UNDER APPLICATION OF MICROWAVES
}

\author{
P. KASTANEK ${ }^{1}$ \\ F. KASTANEK ${ }^{2, *}$ \\ M. HAJEK ${ }^{2}$ \\ J. SOBEK ${ }^{2}$ \\ O. SOLCOVA ${ }^{2}$
}

Received: $10 / 12 / 09$

Accepted: 30/11/10

\author{
${ }^{1}$ Institute of Chemical Technology, \\ Faculty of Environmental Technology, \\ 16000 Prague 6, Technicka 1905, Czech Republic \\ 2 Institute of Chemical Process Fundamentals, \\ Academy of Sciences of the Czech Republic, \\ 16502 Prague 6 -Suchdol, Rozvojova 135, Czech Republic
}

*to whom all correspondence should be addressed: e-mail: Kastanek@icpf.cas.cz

\begin{abstract}
Laboratory-scale experiments were performed in order to examine the effect of microwave field on the effectiveness of KPEG method (nucleophilic substitution of the chlorine atoms in a molecule of PCBs by alkaline polyethylene glycol PEG) in the removal of PCBs from highly contaminated mineral oils with the content of indicator congeners (No 28,52,101,53,138 and 180) above $5500 \mathrm{mg} \mathrm{kg}^{-1}$. Results show that the microwave field significantly increases the reaction rate and the method's effectiveness in the removal of chlorine from the molecules of PCBs. Addition of a small amount of an ionic liquid, in this case 1-butyl-3-methylimidazolium hexafluorophosphate-[bmim][PF6], significantly positively affects the results obtained under the influence of microwave field. The KPEG method enhanced by the application of microwaves could be used in practice for decontamination and recycling of PCB-containing dielectric fluids in electric capacitors and transformers. High efficiencies of the reaction were achieved even when microwaves were applied with no PEG added, only by the presence of alkaline hydroxide or by the presence of a small amount, up to ca. $2 \%$, of an ionic liquid [bmim][PF6]. The microwave enhanced KPEG method might has some advantages for practical uses in decontamination applications.
\end{abstract}

KEYWORDS: PCBs, mineral oil, nucleophilic substitution, polyethylene glycol, $\mathrm{KOH}$, microwaves, ionic liquids.

\section{INTRODUCTION}

If we do not accept incineration as a long term acceptable solution, removal of polychlorinated biphenyls from oils used as dielectric liquids in electric capacitors and transformers remains a pressing problem. In years 1996-2002, pilot plant and industrial scale testing of selected alternative methods of PCBs removal from various media was undertaken in Czech Republic. Results of the tests were summed up by the authors of this paper (Kastanek, 2005). Industrial tests performed with more of $500 \mathrm{~kg}$ of contaminated oils in a batch showed that the known nucleophilic methods (KPEG), in contrast with the published data (Brunelle et al., 1985), destructed PCBs in the oils very slowly, even when high temperatures (around $160^{\circ} \mathrm{C}$ ) were used. Reduction by micro-dispersed metal sodium was relatively faster. This method however creates significant risks of hydrogen release and formation of explosive mixtures even by the presence of traces of water. Moreover, because the reaction takes place above the sodium melting point, the sodium powder sinters and the reaction controlling surface area of the powder decreases. In order to maintain the required reaction rate, it is necessary to work at high shear stresses of the agitator. This is difficult to realize when large industrial reactors are used.

On the other hand, the methods of nucleophilic substitution of the chlorine atoms were proven to be technologically feasible and were tested on both the pilot plant-scale and the industrial-scale 
productions. For lower levels of contamination (up to thousands ppm of PCBs) they were found to be cost-effective.

Therefore the method of nucleophilic substitution of chloroatoms by alkaline polyethylene glycol was chosen for experimental investigation as to whether microwave irradiation can facilitate destruction of PCBs in contaminated oils.

Thus, it was expected that the microwave field would increase the efficiency of PCBs destruction and would lower the reaction times. We assumed that both the thermal and the non thermal effects of microwave field would positively affect the course of the reactions (Hájek, 2006; Jakob et al., 1995; Lidstrom, 2005). It was expected that in the system of two non-miscible phases created by the oil phase and the PEG phase, local overheating in "hot spots" would take place in both phases. This might have positive effects on the parameters of the Arrhenius equation and through them positively influence the reaction rate constant. Additionally, it was hoped that the effect of different absorption of microwaves by the two phases could "catalyze" the interfacial heat and mass transfer and speed up the transport of the reacting compounds. Compared to the classical ways of heating, microwaves have advantages of significantly increased reaction rates, milder reaction conditions, higher yields of chemical reactions, purity of the products, less energy consumption, and increase or modification of reaction selectivity (chemo-, regio- and stereoselectivity). These effects cannot be achieved by using classical heating.

In the mid-eighties a simple and safe method for the removal of PCBs from transformers oils was proposed (Brunelle et al., 1985). The method allowed for subsequent recycling and further utilization of the treated transformer oil. It was found that polyethylene glycol (PEG) and poly-(ethylene glycol methyl ether) (PEMG) can act as catalysts and nucleophiles when reacting with PCBs in alkaline environment at relatively low temperatures around $60-120^{\circ} \mathrm{C}$ in non- polar solvents like transformer oils. It was reported that the reaction is relatively fast and complete degradation is achieved in 2 hours. The reaction products are partially or fully dehalogenated biphenyl polyglycols (substituted glycol ethers). Because the products are present in the PEG phase, they can be easily separated from the transformer oil.

Potassium hydroxide and PEG 300-500 are the most commonly used reagents for this reaction. Potassium polyethylene glycolate, which is a potent nucleophilic agent and can attack the $\mathrm{C}-\mathrm{Cl}$ bond even at the earlier mentioned mild temperatures, is formed by the reaction of PEG with $\mathrm{KOH}$.

It seems that the reaction does not show regio-selectivity, nevertheless congeners with higher number of chlorine atoms (ideally with 6-7 atoms) are degraded preferentially. Both laboratory and pilot plant tests performed with less chlorinated PCBs differ significantly (Kastanek, 2005) from the original data (Brunelle et al.,1985)) and indicate that the reaction rate of congeners with three and four chlorine atoms is much lower (Sabata et al.,1993). This is important for example for decontamination of transformer oils in Czech Republic, where the technical mixture DELOR 103, containing mainly less chlorinated congeners was used widely in dielectric fluids. Treatment of less chlorinated PCBs by the KPEG method requires large volumes of reagents and temperatures much higher than $100^{\circ} \mathrm{C}$ in the range of $145-170^{\circ} \mathrm{C}$ (Kastanek, 2005). However at these temperatures we are already crossing the line of safe work environment because molecular hydrogen is being released and the reaction is very difficult to be controlled.

Another problem associated with KPEG and its modifications, still open to investigation, is the possibility of creating dangerous toxic reaction intermediates. Longer heating of aryl polyglycols (produced by the reaction of PCBs with the alkaline glycolate) by the presence of a base leads via elimination of a vinyl-terminated PEG to the formation of corresponding polychlorobiphenyl alcohols which are known precursors of polychlorinated dibenzofuranes (Choudhary et al., 1983). It is hoped that these negative aspects of KPEG could be suppressed by applying microwave technique.

\section{METHODS}

\subsection{Concentration and composition of PCBs in the liquid}

The oil which was used for the experiments was taken from a power factory capacitor that was manufactured by ZEZ Zamberk, Czech Republic. The oil was contaminated with the technical mixture DELOR 103, composed mostly by the less chlorinated PCBs, which account for almost $90 \%$ of all congeners in the mixture ( $($ PCB 5,8 $=13.6 \%, 15,18=11.5 \%, 17=8.8 \%, 16,32=13.4 \%, 26=$ $1.6 \%, 31=7 \%, 28=15.1 \%, 20,33,53=8.5 \%, 22,51=4.9 \% 52=1.8 \%, 49=1.7 \%, 101=0.17 \%)$. In accordance with the directives of the Ministry of the Environment of the Czech Republic, the extent of the contamination of the environment by PCBs is judged by the presence of so-called 
indicator congeners. Out of the existing 209 PCB congeners, seven were selected to represent the spectrum of congeners in the technical mixtures that were most often used in the former Czechoslovakia. The sum of the concentrations of the indicator congeners is used to define the allowable residual concentrations of PCBs in decontaminated materials that are prescribed by the law. The concentration of the indicator PCBs (Nos. 28, 52, 101, 153, 138 a 180) determined before the reaction was $5566 \mathrm{mg} \mathrm{L}^{-1}$. Concentrations of the indicator congeners around $5000 \mathrm{mg} \mathrm{L}^{-1}$ were typical for the so-called "oil capacitors" whose dielectric filling contained mostly mineral oils with lower concentrations of PCBs. Concentrations lower than $5000 \mathrm{mg} \mathrm{L}^{-1}$ of the indicator congeners may be also found in mineral oils that were used to rinse remnants of PCBs from capacitors and transformers.

\subsection{Analysis of $P C B s$}

Gas chromatography on a capillary column with chemically bonded non-polar silicone phase and controlled temperature profile was used. The HP $5 \mathrm{MS}$ column was $60 \mathrm{~m}$ long, had a $0.25 \mathrm{~mm}$ internal diameter and was carrying a film of stationary phase $0.25 \mu \mathrm{m}$ thick. The stationary phase contained 5\% biphenyl and 95\% dimethylsiloxane.

Nitrogen was used as the mobile phase. The inlet pressure of nitrogen was $140 \mathrm{kP}$ and the flow rate was $1 \mathrm{~mL} \cdot \mathrm{min}^{-1}$. A $1 \mu \mathrm{L}$ sample was injected in splitless injection mode. The column was equipped with the ECD detector. The detector temperature was set at $300^{\circ} \mathrm{C}$ and the gas flow rate through the detector was $30 \mathrm{~mL} \mathrm{~min}^{-1}$. The chromatographs were evaluated with the help of the known retention times. A solution of the standard PCB MIX was used for the evaluation of the individual indicator congeners $(28,52,101,138,153,180)$. The analyses were performed in the Institute of Chemical Technology in Prague (Faculty of Environmental Technology, Prof. J. Burkhard).

\subsection{Chemicals}

Polyethylene glycol 575, Aldrich (45, 90-3).

Potassium hydroxide, pellets, 85\%, Aldrich (22,147-6).

Ionic liquid: 1-butyl-3 methylimidozolium hexafluorophosphate [bmim][PF6]

\subsection{Microwave reactor}

Laboratory microwave Labstation for Synthesis MICROSYNTH, Milestone, 2 magnetrons $1000 \mathrm{~W}$, was used. On the top of the oven is an opening, $30 \mathrm{~mm}$ in diameter, with a Teflon sealing ring. A glass condenser allowing outgoing vapor to condense protrudes through the opening. The condenser is connected to a $250 \mathrm{~mL}$ boiling flask, which resides inside the oven's cavity and serves as the reactor. Small batches of the reaction mixture were mixed by a magnetic stirrer located under the bottom of the cavity. Larger volumes were agitated by a glass impeller. Impeller's blades were collapsible to allow their passage through a shaft aperture into the reactor. A dosing pipette was also placed into the aperture and sealed by a Teflon gasket.

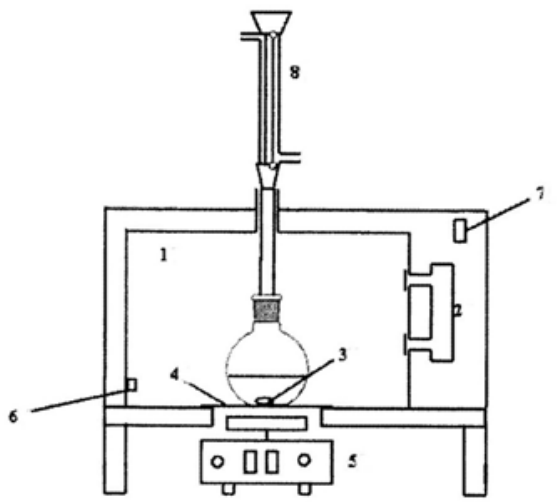

Figure 1. Chart of experimental set up

1-cavity 2; 2-magnetron, generator of the microwave field; 3-magnetic stirring bar; 4-aluminum cavity bottom; 5 - magnetic stirrer with the control of revolution speed; 6-IR temperature sensor; 7-main switch; 8- Liebig condenser 


\subsection{Alkaline decomposition of PCBs in oils by KPEG method without using microwaves}

The choice of the nucleophilic reactant composition was influenced by our previous experimental results (Kastanek, 2005) and by our experience with reaction rates of the decomposition of the lower chlorinated PCB congeners.

Contaminated oil (sample $\mathrm{K}-0$ ) in $30 \mathrm{~mL}$ quantity was poured into the reactor which was equipped with the condenser and magnetic stirrer and the reactor was placed on a hot plate.

The sample was heated to an initial temperature of $125^{\circ} \mathrm{C}$ and then the individual components of the reaction agent were added. Several combinations of the amounts of PEG 575 and powdered KOH were tested. PEG 575 was added in the following amounts: $10 \mathrm{~g}, 20 \mathrm{~g}, 30 \mathrm{~g}$. Powdered $\mathrm{KOH}$ (approximately $15 \%$ moisture) was added in amounts $1.5 \mathrm{~g}, 3 \mathrm{~g}$, and $4.5 \mathrm{~g}$. The constant ratio $\mathrm{KOH} /$ PEG $=0.15$ was selected based on the results of our previous work (Kastanek, 2005).

The sample was maintained at the temperature $125-145^{\circ} \mathrm{C}$ for 30,60 , and 90 minutes. The reaction took place in the atmospheric air. The mixture was vigorously agitated during the entire reaction time. After the reaction was completed, the mixture was quickly cooled in the freezer to a temperature bellow $8^{\circ} \mathrm{C}$, the oil phase was separated and handed over for the analysis (samples $\mathrm{K}$ 1). The sample which contained $30 \mathrm{~mL}$ of oil, $20 \mathrm{~g}$ of PEG 575 , and $3 \mathrm{~g}$ of $\mathrm{KOH}$ (see results) $1.0 \mathrm{~g}$ of ionic liquid [bmim] [PF6] was added. At the end of the reaction, the mixture was quickly cooled in the freezer below $8^{\circ} \mathrm{C}$, the oil phase was separated and handed over for the analysis (samples $\mathrm{K}-2$ ).

\subsection{Alkaline decomposition of PCBs in oils by KPEG method enhanced by the application of microwave field}

Contaminated oil, mixed with other reactants, was placed in the reactor in the same way as in the experiments performed without using microwaves and described above. The microwave field was applied on the sample which was containing $30 \mathrm{~mL}$ of the contaminated oil, $20 \mathrm{~g}$ of PEG 575, and $3 \mathrm{~g}$ of $\mathrm{KOH}$.

Ionic liquids are very effective absorbents of microwave radiation, therefore it was necessary to coordinate and control the intensity of the microwave field and the duration of the irradiation in order to prevent undesirable overheating of the sample. The used ionic liquid decomposed at temperatures above $185^{\circ} \mathrm{C}$. To prevent the decomposition, the intensity of the microwave field was kept in the range of $300-600 \mathrm{~W}$ and the periods of the irradiation lasted between $30 \mathrm{~s}$ and $120 \mathrm{~s}$. The choice of the intensity and periods of its application was the result of preliminary experiments and previous experience. Based on this information, an optimal algorithm of intermittent application of the microwave field was developed and used. Under intensive mixing of the reaction mixture, the microwave heating was applied for 2 min at the intensity of the microwave field of $440 \mathrm{~W}$. During this time, the mixture temperature climbed all the way to the boiling point; the microwave field was therefore interrupted and the mixture was allowed to cool down for $3 \mathrm{~min}$. Another application of the microwaves for two minutes at the intensity of $440 \mathrm{~W}$ followed by three minutes of cooling. Lastly, the microwaves were applied for one minute at $440 \mathrm{~W}$. The total time of microwave field application was $5 \mathrm{~min}$, the total reaction time, including the breaks for cooling, was $12 \mathrm{~min}$. At the end of the reaction, the mixture was rapidly cooled in a freezer to a temperature below $8^{\circ} \mathrm{C}$, the oil phase was separated and handed over to be analyzed (sample K-3).

One more experiment in the microwave field was performed with the addition of $1.0 \mathrm{~g}$ of ionic liquid $[\mathrm{bmim}]\left[\mathrm{PF}_{6}\right]$ into the reaction mixture. When the microwave field at $440 \mathrm{~W}$ was applied for 1 min 40 $\mathrm{S}$, the temperature of the mixture reached $185^{\circ} \mathrm{C}$ and the ionic liquid partially started to break down, which was manifested by strong ammoniac odor. The periods of microwaves application were then shortened to 30 seconds. During the entire time the mixture was vigorously mixed with a glass agitator. The total time of the microwave application was $5 \mathrm{~min}$, the total time of the reaction, including the cooling breaks, was $12 \mathrm{~min}$. After the reaction stopped, the mixture was rapidly cooled in a freezer to a temperature below $8^{\circ} \mathrm{C}$, the oil phase was separated and handed over to be analyzed (sample K-4).

\subsection{Alkaline decomposition of PCBs in oils with the addition of potassium hydroxide, without PEG}

Two experiments were carried out with no PEG present. Only $1.0 \mathrm{~g}$ of potassium hydroxide was added to the contaminated oil. The experiments were carried out under the same conditions as the experiments that yielded the analytical samples K-1 (no microwaves) and K-3 (with microwaves). The experiments were performed in order to find out whether the reaction between the chlorine in 
the molecules of PCB congeners and the potassium from the hydroxide takes place even in the absence of PEG. This would be beneficial because it happens in some cases, for unknown reasons, it is difficult to separate the decontaminated oil phase from the PEG phase (Kastanek, 2005). The decontaminated oil may then contain too much of the PEG phase and cannot be recycled.

Resulting samples were marked $\mathrm{K}-5$ and $\mathrm{K}-6$ respectively.

The algorithm for applying the microwave field was modified by the addition of two more periods of microwaving, each lasting $1 \mathrm{~min}$. The total time of the microwave field application therefore was 7 $\mathrm{min}$ ). When the reaction stopped, the mixture was cooled and analyzed (sample K-6).

\subsection{Alkaline decomposition of PCBs in oils with the addition of potassium hydroxide, without PEG, in the presence of an ionic liquid}

The experiment with the microwave field was repeated under the same conditions as in the case of the sample $\mathrm{K}-6$, with $1 \mathrm{~g}$ of ionic liquid [bmim][PF6] added to $30 \mathrm{~mL}$ of contaminated oil. When the reaction stopped, the reaction mixture was cooled and analyzed (sample K-7)

\section{RESULTS}

Using a higher amount of PEG did not pay off because it resulted in only a slight increase in the amount of decomposed PCB's.

Regarding reaction times, 30 minutes was not long enough; the highest dehalogenation percentage at this reaction time was $21 \%$. The reaction time of 90 minutes did not result in a satisfactory dehalogenation percentage either; the maximum dehalogenation reached at this longer time (and logically higher energy expenditure) amounted to 66\%.

Table 1 reports the results of experiments with KPEG and $\mathrm{KOH}$ (20g of PEG 575 and $3 \mathrm{~g}$ of $\mathrm{KOH}$ ) without the application of the microwave field and with the application of the microwave field. It also shows the effects of the ionic liquid.

Table 1. Dehalogenation of oils by methods KPEG, and $\mathrm{KOH}$

without and with the application of the microwave field, for different reaction times, $\mathrm{t}$

\begin{tabular}{lrrr}
\hline \multicolumn{1}{c}{ Hample } & $\begin{array}{r}\text { Concentration } \text { PCB }^{1} \\
\left(\mathrm{mg} \mathrm{L}^{-1}\right)\end{array}$ & $\begin{array}{r}\text { Dehalogenation } \\
(\%)\end{array}$ & $\begin{array}{r}t \\
\text { (minutes) }\end{array}$ \\
\hline K-0 & 5566 & - & - \\
\hline K-1 (KPEG, without microwaves) & 2469 & 55,64 & 60 \\
\hline K-2 (KPEG, without microwaves, & & & \\
[bmim][PF6]) & 2129 & 61,74 & 60 \\
\hline K-3 (KPEG, with microwaves) & 9,27 & 99,83 & 12 \\
\hline K-4 (KPEG, with microwaves, & & & \\
{$[$ bmim][PF6]) } & 0,195 & 99,996 & 12 \\
\hline K-5 (KOH, without microwaves) & 4860 & 12,68 & 60 \\
\hline K-6 (KOH, with microwaves) & 586 & 89,47 & 7 \\
\hline K-7 (KOH, with microwaves, & & & \\
{$[$ bmim][PF6]) } & 87 & 98,44 & 7 \\
\hline
\end{tabular}

${ }^{1}$ Concentration of the six indicator congeners. The results were calculated as an arithmetic average of three measurements. The individual measurements differ less then $0 \pm 18 \%$.

It is obvious that the microwave application has a significant impact on shortening the reaction time and on reducing the final concentrations of PCBs in the oil to the level bellow $10 \mathrm{mg} \mathrm{kg}^{-1}$. In cases when the ionic liquid was added in the amount of $2 \%$ of the mixture, the dehalogenation efficiency improved to the levels over $99.99 \%$ after merely 5 minutes of the microwave application; the final concentrations of PCBs in the oil were deeply below $1 \mathrm{mg} \mathrm{kg}^{-1}$. As far as the experiments performed with only potassium hydroxide, the results are noteworthy. It turns out that this method could find use in practice during decontaminations of dielectric liquids from electric capacitors or transformers. The method has high enough efficiency and the alkalinity is not an obstacle because the old dielectric fillers have usually higher acidity which could be neutralized by the residual hydroxide. The increase in the efficiency of the KPEG method that was brought about by the application of microwaves is likely due to the thermal effects of the microwaves that were mentioned in the introduction. As for any non-thermal effects of the microwave field, it is possible to speculate that non-polar PCBs have 
some microwave absorption capacity and that the microwaves force the chlorine-carbon bonds on the aromatic ring to oscillate. The excited bonds are more accessible to nucleophilic substitution by the KPEG reagent. The action of the microwave field on this two-phase reaction mixture, whose two phases have different affinity for absorption of microwaves, may be regarded as a kind of "catalysis" of the interfacial mass transfer with chemical reaction. The presence of solid $\mathrm{KOH}$ in the contaminated oil brings about massive absorption of the microwaves; this most likely leads to the overheating of the interfacial area and to the acceleration of the reaction.

\section{CONCLUSIONS}

Enhancement of the KPEG method by applying microwaves brings about acceleration of the dehalogenation reaction-after 5 minutes of the microwave action, the efficiency of PCB degradation reaches 99\%. A similar experiment with conventional heating requires 60 minutes to obtain efficiency around 55-60\%. The chromatograms show that even less chlorinated PCB congeners which have 3-4 atoms of chlorine in a molecule are degraded rapidly when microwaves are applied. The use of ionic liquids as a reaction medium component in microwave-enhanced degradation of PCBs although, to our best knowledge, not mentioned in the literature, is very interesting. Applications of ionic liquids represent modern trends of today's chemical technology. Additions of very small amounts (within 1\% of the reaction mixture volume) result in intensive absorption of microwaves and acceleration of the dehalogenation reaction. It is unclear whether the ionic liquid acts merely as an absorbent of the microwave radiation or, in a given case also participates in the reaction. The question of further optimization of the reaction conditions when using ionic liquids, especially the determination of the optimal amount of the ionic liquid, also remains open. For example, addition of $2 \%$ of ionic liquid $\left[\mathrm{bmim}\left[\mathrm{PF}_{6}\right]\right.$ in the reaction mixture when classical heating was used led to only small increase of the dehalogenation efficiency (from $55 \%$ to $61 \%$ ).

The results obtained here are of fundamental importance for the design of industrial processes for simple and economic degradation of PCBs from contaminated oils in a continuous microwave reactor with the addition of traces of ionic liquids. Further experimental data are necessary to enable more profound understanding of the reaction kinetic and the reaction mechanism. Because the used chemicals, including ionic liquids, collect in the phase that is immiscible with the oil, it is expected that the decontaminated oils may be recycled. Their dielectric properties are more or less preserved considering the short times they are exposed to higher temperature during the decontamination. In practical applications where recycling of the decontaminated oil is not required, the method of PCBs degradation using potassium hydroxide alone, with no PEG present, shows promising for further exploring.

\section{ACKNOWELEDGEMENT}

This work was supported by the Grant agency of the Czech Republic 104/09/0694 and by grant of Ministry of Environment of the Czech Republic SP/2f3/133/08.

\section{REFERENCES}

Brunelle D.J., Mendiratta A.K. and Singleton D.A. (1985), Reaction/removal of polychlorinated biphenyls from transformer oil: treatment of contaminated oil with poly(ethylene glycol)/KOH, Environ. Sci. Technol., 19, 740-746.

Choudhary G., Keith L.H. and Rappe C. (1983), In: Chlorinated Dioxins and Dibenzofurans in the Total Environment, Butterworth: Boston, MA.,US.

Hájek M. (2006), Microwave catalysis in organic synthesis, Loupy A., Ed., pp. 615-652. Wiley-VCH.

Jakob J., Chia L.H.L. and Boey F.Y.C. (1995), Thermal and nonthermal interaction of microwave radiation with material, J. Mat. Sci., 30, 5321-5327.

Kastanek F. and Kastanek P. (2005), Combined decontamination processes for wastes containing PCBs, J. Hazard. Mater., B117, 185-205.

Sabata S., Friesova A., Rericha R.and Hetflejs J. (1993), Limits to the use of KOH/PEG method for destruction of PCB liquids of Czechoslovak production, Chemosphere, 27, 1201-1210.

Tierney J.P and Lidstrom P., Eds. (2005), Microwave assisted organic synthesis, Blackwell Publishing, Oxford, UK. 